\title{
Evaluation of paradoxical vocal cord motion and differential diagnosis
}

\author{
Vokal kordun paradoks motilitesinin ve ayırıcı tanısının değerlendirmesi \\ Esin Yalçınkaya', İsmail Güler², Sinan Kocatürk² \\ ${ }^{1}$ Yiiksek İhtisas University Koru Hospital, Ankara, Turkey \\ ${ }^{2}$ Department of Otorbinolaryngology, Faculty of Medicine, Ufuk University, Ankara, Turkey
}

\begin{abstract}
Objective: To evaluate the relationship between paradoxical vocal cord motion (PVCM) disease and the diseases such as asthma, laryngopharyngeal reflux and anxiety disorder that should be taken into consideration most frequently in differential diagnosis.

Methods: The study included 100 patients (64 females and 36 males) who had applied to the polyclinics of pulmonary diseases, gastroenterology, psychiatry and ear nose throat due to respiratory symptoms. In asymptomatic period, it was planned to diagnose paradoxical vocal cord motion due to inspiratory adduction and posterior glottic fissure observed in videolaryngoscopic examination made after provocative maneuvers.

Results: The mean age of the patients was $44.7 \pm 7.3$ (female: $36.3 \pm 4.5$, male: $52.3 \pm 3.2$ ). $57 \%$ of the patients applied to pulmonary diseases, $24 \%$ to gastroenterology, $12 \%$ to psychiatry and $7 \%$ to ENT polyclinics. No paradoxical vocal cord motion was detected in 99 patients invideolaryngoscopic examination made after provocative maneuvers performed in asymptomatic period. Paradoxical vocal cord motion was detected in one patient who had attack during videolaryngoscopic examination.

Conclusion: Although PVCM is a rarely seen disease, it leads to serious problems in non-diagnosed patients. In our study, we concluded that PVCM can be diagnosed during an attack rather than provocativemaneuvers performed during asymptomatic period.
\end{abstract}

Keywords: Paradoxical vocal cord motion, asthma, laryngopharyngeal reflux, anxiety disorder.

Paradoxical vocal cord motion (PVCM) is defined as closure of vocal cords improperly in inspirium..$^{[1]}$ The patients generally have obstructive airway complaints such as stridor, wheezing, dyspnea and cough while breathing. ${ }^{[1,2]}$ As it is a rarely seen functional disorder, generally misdiagnoses and treatments are made. ${ }^{[2,3]}$ PVCM is basically diagnosed by

\section{Özet}

Amaç: Çalışmanın amacı, paradoks vokal kord motilitesi hastalığının, ayırıcı tanıda en büyük sıklıkla hesaba katılması gereken astım, larengogarengeal reflü ve anksiyete bozukluğu ile ilişkisini değerlendirmektir.

Yöntem: Bu çalışmaya akciğer hastalıkları, gastroenteroloji, psikiyatri ve respiratuar semptomlar nedeniyle kulak burun boğaz polikliniklerine başvuran 100 (64 kadın ve 36 erkek) hasta dahil edilmiştir. Asemptomatik dönemde inspiratuar addüksiyona bağlı paradoks vokal kord motilitesine ve provokatif manevralar sonrasında videolarengoskopik muayenede gözlemlenen posterior glotik fissüre tanı konması planlanmıştır.

Bulgular: Hastaların yaş ortalaması 44.7 \pm 7.3 (kadın: $36.3 \pm 4.5$, erkek: $52.3 \pm 3.2)$ idi. Hastaların \% 57'si akciğer hastalıkları, \%24'ü gastroenteroloji, \% 12'si psikiyatri ve \% 7'si KBB polikliniklerine başvurmuştu. Asemptomatik dönemde provokatif manevralar sonrası yapılan videolarengoskopik muayenede 99 hastada paradoks vokal kord motilitesi saptanmamıştır. Videolarengoskopik muayene sırasında atak oluşan bir hastada paradoks vokal kort motilitesi saptanmıştır.

Sonuç: Paradoks vokal kord motilitesi nadiren görülen bir hastalık olmasına ră̆men tanı konmamış hastalarda ciddi sorunlara yol açar. yol açar. Çalışmamızda, bu hastalığın tanısının, asemptomatik dönemde gerçekleştirilen provokatif manevralardan ziyade, atak sırasında konabileceği sonucuna vardık.

Anahtar sözcükler: Paradoks vokal kord motilitesi, astım, larengofarengeal reflü, anksiyete bozukluğu.
Correspondence: Esin Yalçınkaya, MD. Yüksek İhtisas University Koru Hospital, Ankara, Turkey.

e-mail: esinkbbesin@gmail.com

Received: April 10, 2014; Accepted: May 17, 2014
Online available at: www.jmedupdates.org doi:10.2399/jmu.2014003004 QR code: adduction of vocal cords in inspirium and observation of a rectangular posterior glottic fissure during laryngoscopic examination during an attack. ${ }^{[3,4]}$ However, there are publications notifying that the disease can be detected by applying provocative maneuvers which induce paradoxical vocal cord motions in the patients during asymptomatic period. ${ }^{[3,5,6]}$ 
It has been reported that PVCM can be confused with laryngopharyngeal reflux, asthma and anxiety disorders and it can be also triggered by these diseases. The present study investigated the prevalence of PVCM in the society and its relationships with asthma, laryngopharyngeal reflux and anxiety disorders.

\section{Materials and Methods}

The study protocol was approved by the ethics committee of our university. Informed consent forms were taken from all patients. The study population was composed of the patients who had applied to pulmonary diseases, gastroenterology, psychiatry or ear nose throat (ENT) outpatient clinics due to vocal changes or respiratory symptoms. Among these patients, those with vocal changes, respiratory disorders, chronic coughs, episodic dyspnea, wheezing, inspiratory stridor symptoms or findings were directed to ENT polyclinics. Anamnesis and additional patient information of these patients were recorded. The patients with underlying neurologic deficit, history of upper respiratory tract operation which might lead to vocal cord dysfunction or the patients who rejected videolaryngoscopic examination or required mechanical ventilation were excluded from the study. The patients were subjected to videolaryngoscopic examination by ENT department and vocal cord motions were recorded by applying maneuvers, which provoke paradoxical vocal cord motions (deep and fast breathing, holding the breath, sniffling and fonation) during examination. Detection of posterior glottic fissure in inspiratory adduction during laryngoscopic examination was accepted as "paradoxical vocal cord adduction". All patients with paradoxical vocal cord motions were planned to be evaluated by gastroenterology, pulmonary diseases and psychiatry departments. In the selected patient population, it was planned to examine the prevalence of paradoxical vocal cord motions and the relationships between this functional disorder and asthma, laryngopharyngeal reflux and anxiety disorders.

\section{Results}

The study included a total of hundred patients: 36 men and 64 women (Table 1). The mean age of the patients was $44.7 \pm 7.3$ years while mean ages of women and men were $36.3 \pm 4.5$ and $52.3 \pm 3.2$ years, respectively. The patients with asthma $(n=57)$ were referred to department of chest diseases, cases with gastroesophageal reflux $(n=12)$ to gastroenterology and anxiety disorders $(n=12)$ to psychiatry departments. Seven patients with vocal cord dys- function applied to ENT polyclinics (Table 1). Except for asthma, gastroesophageal reflux and psychological disorders no other systemic disorder caused vocal cord dysfunction.

All patients showed one of the symptoms or findings of vocal change $(58 \%)$, respiratory disorder $(62 \%)$, chronic cough (43\%), episodic dyspnea (28\%), wheezing (13\%) and inspiratory stridor (7\%) (Table 1). Only one patient had asthma attack during videolaryngoscopic examination who was monitored during the attack. In endoscopic larynx examination, radix linguae and laryngeal regions of all patients were normal. When evaluated in terms of vocal cord function, inspiratory adduction and posterior glottic fissure were detected only in the patient who had attack during examination (Fig. 1). The patients with PVCM were evaluated by pulmonary diseases, gastroenterology and psychiatry departments.

Respiratory function test results and pulmonary findings were normal. After endoscopic examination applied to the patient with dyspeptic complaints, gastroesophageal reflux was diagnosed and the treatment was initiated. During psychiatric examination of the patient, anxiety induced by attacks was detected and use of sedative treatment was planned. Vocal cord functions of all other patients were considered as normal. It was seen that provocative exercises during asymptomatic period did not trigger vocal cord dysfunction.

\section{Discussion}

Larynx is an organ functioning as a valve between trachea and esophagus. ${ }^{[7]}$ The muscle primarily responsible for vocal cords is posterior cricoarythenoid muscle. Adduction is basically ensured with lateral chricoarythenoid muscle. ${ }^{[7,8]}$ Glottic fissure during normal inspirium is controlled by medullar respiratory area by means of vagal nerve. Therefore, vocal cord abduction is ensured with contraction of posterior cricoarythenoid muscles. During normal expiration, tonic activity of the posterior cricoarythenoid muscle decreases and rima glottis constricts $10 \%$ and $40 \%$ with the contraction of lateral cricoarythenoid muscle. This narrowing starts immediately before expiration and continues during approximately $95 \%$ of the expiratory phase. ${ }^{[8,9]}$ It is known that the receptors in respiratory tracts lead to closure of vocal cords and coughing as a part of glottic closing reflex. This reflex is triggered with the stimulants which cause irritation. It is considered that these stimulations in the airways lead to development of glottic closing reflex in the individuals with hypersensitivity. ${ }^{[9-11]}$ 
Paradoxical vocal cord motility was first introduced by Christopher as vocal cord dysfunction. ${ }^{[9]}$ In the literature, it is defined with the terms "paradoxical vocal cord adduction, episodic paroxysmal laryngospasm, irritable larynx syndrome and respiratory dystonia". ${ }^{[12-14]}$ Stimulation of airway receptors is caused by some factors such as laryngopharyngeal reflux, allergy, asthma, psychological disorders, rhinosinusitis and inhalation of irritating substances. ${ }^{[15,16]}$

Organic and non-organic factors were indicated in the etiology of PVCM disease. ${ }^{[17]}$ It was stated that organic reasons are less frequently encountered than non-organic reasons. Organic reasons include brain stem compression, cortical or upper motor neuron damage, gastroesophageal reflux, nuclear or lower motor neuron damage while nonorganic reasons include simulative behaviors and conversion disorders. ${ }^{[18-20]}$ PVCM is most frequently seen in young women. ${ }^{[19]}$ In our study, $64 \%$ of the patients were female and their mean age was $36.3 \pm 4.5$ years.

There are many case reports in the literature about vocal cord dysfunction. These cases are generally related to patients with attacks characterized by respiratory tract symptoms such as long-lasting coughing, dyspnea, vocal change, wheezing and stridor. ${ }^{[1,20,21]}$ The gold standard in the diagnosis of PVCM is monitorization of vocal cords during an attack by means of videolaryngoscopy. ${ }^{[2,23]}$ Some publications have suggested establishment of diagnosis with stimulation of symptoms by provocative exercises during asymptomatic period. ${ }^{[6,2,2,2]}$ In a study performed with asthma patients, Yelken et al. stimulated vocal cord motions during asymptomatic period with attack simulation and various maneuvers. They stated that 20 of 96 patients had PVCM ${ }^{[6]}$ Some authors have concluded that PVCM cannot be diagnosed with provocative exercises performed during asymptomatic period but it can be diagnosed during laryngoscopic examination made at the time of an attack or during exercise. ${ }^{[24,25]}$ Heimdal et al. developed 'continuous laryngoscopy' technique applied during treadmill exercise and stated that it was useful in establishment of the diagnosis of PVCM during asymptomatic period. ${ }^{[25]}$ In our study, 99 of 100 patients were subjected to provocation maneuvers during asymptomatic period, however any PVCM was not encountered. In one of our patients who had attack during the examination, inspiratory adduction and posterior glottic fissure were detected at the time of vocal cord motions.

In differential diagnosis, asthma, laryngopharyngeal reflux and anxiety disorders should be taken into consideration. ${ }^{[2,27]}$ The symptoms of asthma include wheezing, dyspnea, feeling of pulmonary stress and cough as a result of immediate narrowing of small airways in the lungs.

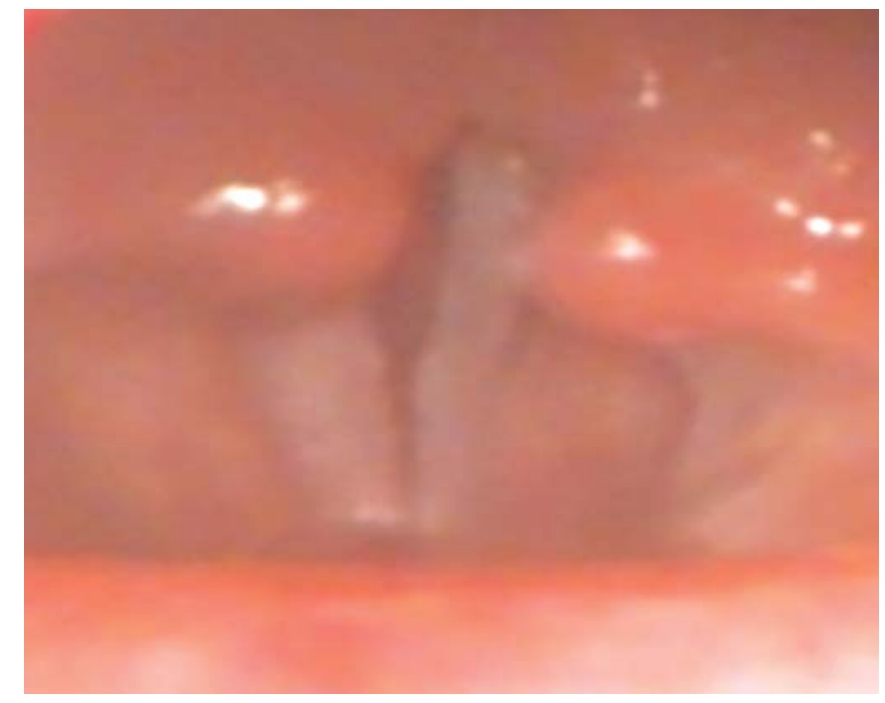

Fig. 1. Videolaryngoscopic image of the patient with paradoxical vocal cord motion at the time of attack. Inspiratory adduction, posterior glottic fissure.

While beta agonist medication is effective in the recovery of asthma symptoms, it is not effective on PVCM symptoms. ${ }^{[28]}$ It is considered that gastroesophageal reflux is one of the most frequent reasons, which trigger PVCM disease. Contact of stomach contents with laryngopharyngeal structures may lead to increased parasympathetic activity in internal laryngeal muscles and paroxysmal attacks. In a dog study, it was stated that a $\mathrm{pH}$ value lower than or equal to 2.5 had sensitized chemoreceptors in laryngeal mucosa and laryngospasm had occurred with the stimulation of the vagal nerve. ${ }^{[26]}$ It is known that anxiety and emotional stresses do also trigger PVCM diseases. It was stated that the patients with PVCM disease are perfectionist, ambitious individuals with high anxiety level. ${ }^{[27]} \mathrm{In}$ our study, $57 \%$ of the patients had asthma, $24 \%$ gastroesophageal reflux and $12 \%$ anxiety disorders.

Treatment methods include application of heliox, nebulized lignocain, anticholinergic inhalation, positive air pressure, sedative and anxiolytic agents at the time of the attack. ${ }^{[2,29]}$ In some publications it has been indicated that psychotherapy, talking therapies and injection of botilinium toxin can be useful chronic treatment modalities. ${ }^{[30,31]}$ The asthma treatment applied to our patient with PVCM disease was given up and talking therapy (6 sessions) was initiated in addition to a stomach protective therapy (proton pump inhibitor) and a sedative agent (serotonin receptor antagonist). It was reported that attacks of the patient did not recur during the 6-month follow-up period. 
Table 1. Demographic and clinic information of the patients included in the study $(n=100 ; 64 F$ and $36 \mathrm{M})$.

\begin{tabular}{|c|c|c|c|c|c|}
\hline Age & Gender & Symptom & Relevant department & Laryngeal examination & Diagnosis \\
\hline 45 & $\mathrm{~F}$ & Vocal change, respiratory disorder & Pulmonary diseases & Normal & NVCM \\
\hline 53 & $\mathrm{~F}$ & Respiratory disorder, episodic dyspnea, wheezing, inspiratory stridor & Pulmonary diseases & Normal & NVCM \\
\hline 28 & M & Vocal change, respiratory disorder, chronic cough & Pulmonary diseases & Normal & NVCM \\
\hline 65 & M & Respiratory disorder, chronic cough & Pulmonary diseases & Normal & NVCM \\
\hline 53 & $\mathrm{~F}$ & Respiratory disorder, episodic dyspnea, wheezing & Pulmonary diseases & Normal & NVCM \\
\hline 18 & $\mathrm{~F}$ & Respiratory disorder, episodic dyspnea, wheezing & Pulmonary diseases & Normal & NVCM \\
\hline 44 & M & Respiratory disorder & Pulmonary diseases & Normal & NVCM \\
\hline 26 & $\mathrm{~F}$ & Vocal change, respiratory disorder & Pulmonary diseases & Normal & NVCM \\
\hline 72 & $\mathrm{~F}$ & Respiratory disorder, episodic dyspnea, wheezing & Pulmonary diseases & Normal & NVCM \\
\hline 65 & M & Respiratory disorder, episodic dyspnea & Pulmonary diseases & Normal & NVCM \\
\hline 47 & M & Vocal change, chronic cough & Pulmonary diseases & Posterior laryngitis & LFR \\
\hline 38 & $\mathrm{~F}$ & Respiratory disorder, wheezing & Pulmonary diseases & Normal & NVCM \\
\hline 31 & $\mathrm{~F}$ & Respiratory disorder, episodic dyspnea, wheezing & Pulmonary diseases & Normal & NVCM \\
\hline 33 & M & Vocal change, chronic cough & Pulmonary diseases & Normal & NVCM \\
\hline 27 & $\mathrm{~F}$ & Respiratory disorder, episodic dyspnea & Pulmonary diseases & Normal & NVCM \\
\hline 58 & M & Respiratory disorder, episodic dyspnea, inspiratory stridor & Pulmonary diseases & $\begin{array}{l}\text { Inspiratory adduction, } \\
\text { Posterior glottic fissure }\end{array}$ & PVCM \\
\hline 29 & $\mathrm{~F}$ & Episodic dyspnea, inspiratory stridor & Pulmonary diseases & Normal & NVCM \\
\hline 31 & M & Respiratory disorder, episodic dyspnea & Pulmonary diseases & Normal & NVCM \\
\hline 48 & $\mathrm{~F}$ & Respiratory disorder, chronic cough & Pulmonary diseases & Normal & NVCM \\
\hline 42 & $\mathrm{~F}$ & Respiratory disorder, episodic dyspnea, inspiratory stridor & Pulmonary diseases & Normal & NVCM \\
\hline 29 & M & Chronic cough & Pulmonary diseases & $\begin{array}{l}\text { Posterior Laryngitis, } \\
\text { Interarythenoid hyperplasia }\end{array}$ & LFR \\
\hline 37 & $\mathrm{~F}$ & Respiratory disorder & Pulmonary diseases & Normal & NVCM \\
\hline 45 & M & Episodic dyspnea & Pulmonary diseases & Normal & NVCM \\
\hline 53 & $\mathrm{~F}$ & Vocal change, respiratory disorder, chronic cough & Pulmonary diseases & Normal & NVCM \\
\hline 28 & M & Respiratory disorder, episodic dyspnea, inspiratory stridor & Pulmonary diseases & Normal & NVCM \\
\hline 65 & $\mathrm{~F}$ & Respiratory disorder, episodic dyspnea & Pulmonary diseases & Normal & NVCM \\
\hline 53 & $\mathrm{~F}$ & Episodic dyspnea, inspiratory stridor, wheezing & Pulmonary diseases & Normal & NVCM \\
\hline 18 & $\mathrm{~F}$ & Wheezing & Pulmonary diseases & Normal & NVCM \\
\hline 44 & M & Inspiratory stridor & Pulmonary diseases & Normal & NVCM \\
\hline 26 & $\mathrm{~F}$ & Vocal change & Pulmonary diseases & Posterior laryngitis & LFR \\
\hline 72 & M & Respiratory disorder & Pulmonary diseases & Normal & NVCM \\
\hline 65 & $\mathrm{~F}$ & Respiratory disorder, episodic dyspnea, inspiratory stridor & Pulmonary diseases & Normal & NVCM \\
\hline 47 & M & Respiratory disorder & Pulmonary diseases & Normal & NVCM \\
\hline 38 & $\mathrm{~F}$ & Episodic dyspnea, wheezing & Pulmonary diseases & Normal & NVCM \\
\hline 31 & $\mathrm{~F}$ & Respiratory disorder & Pulmonary diseases & Normal & NVCM \\
\hline 33 & M & Vocal change, respiratory disorder, chronic cough & Pulmonary diseases & Normal & NVCM \\
\hline 27 & M & Respiratory disorder, episodic dyspnea & Pulmonary diseases & Normal & NVCM \\
\hline 58 & $\mathrm{~F}$ & Episodic dyspnea & Pulmonary diseases & Normal & NVCM \\
\hline 29 & $\mathrm{~F}$ & Chronic cough & Pulmonary diseases & Normal & NVCM \\
\hline 31 & M & Respiratory disorder, inspiratory stridor & Pulmonary diseases & Normal & NVCM \\
\hline 48 & $\mathrm{~F}$ & Vocal change, respiratory disorder & Pulmonary diseases & Normal & NVCM \\
\hline 42 & M & Respiratory disorder, episodic dyspnea, inspiratory stridor & Pulmonary diseases & Normal & NVCM \\
\hline 29 & M & Respiratory disorder & Pulmonary diseases & Normal & NVCM \\
\hline 23 & $\mathrm{~F}$ & Respiratory disorder & Pulmonary diseases & Normal & NVCM \\
\hline 46 & $\mathrm{~F}$ & Vocal change, respiratory disorder, chronic cough & Pulmonary diseases & Normal & NVCM \\
\hline 37 & $\mathrm{~F}$ & Wheezing & Pulmonary diseases & Normal & NVCM \\
\hline 33 & $\mathrm{~F}$ & Vocal change, respiratory disorder & Pulmonary diseases & Normal & NVCM \\
\hline 34 & $\mathrm{~F}$ & Respiratory disorder, episodic dyspnea, inspiratory stridor & Pulmonary diseases & Normal & NVCM \\
\hline 63 & $\mathrm{~F}$ & Respiratory disorder & Pulmonary diseases & Normal & NVCM \\
\hline 62 & M & Episodic dyspnea & Pulmonary diseases & Normal & NVCM \\
\hline 32 & M & Chronic cough & Pulmonary diseases & Normal & NVCM \\
\hline 38 & $\mathrm{~F}$ & Vocal change, respiratory disorder & Pulmonary diseases & Posterior laryngitis & LFR \\
\hline 35 & $\mathrm{~F}$ & Respiratory disorder & Pulmonary diseases & Normal & NVCM \\
\hline
\end{tabular}


Table 1 [continued]. Demographic and clinic information of the patients included in the study ( $\mathrm{n}=100 ; 64 \mathrm{~F}$ and $36 \mathrm{M})$.

\begin{tabular}{|c|c|c|c|c|c|}
\hline Age & Gender & Symptom & Relevant department & Laryngeal examination & Diagnosis \\
\hline 44 & $\mathrm{~F}$ & Episodic dyspnea & Pulmonary diseases & Normal & NVCM \\
\hline 47 & $\mathrm{~F}$ & Vocal change, respiratory disorder, chronic cough & Pulmonary diseases & Normal & NVCM \\
\hline 37 & $\mathrm{~F}$ & Vocal change, respiratory disorder, chronic cough & Pulmonary diseases & Normal & NVCM \\
\hline 45 & M & Episodic dyspnea & Pulmonary diseases & Normal & NVCM \\
\hline 48 & $\mathrm{~F}$ & Vocal change, respiratory disorder, chronic cough & Pulmonary diseases & Normal & NVCM \\
\hline 39 & $\mathrm{~F}$ & Respiratory disorder & Pulmonary diseases & Normal & NVCM \\
\hline 37 & M & Chronic cough & Gastroenterology & Normal & NVCM \\
\hline 53 & $\mathrm{~F}$ & Vocal change, chronic cough & Gastroenterology & $\begin{array}{l}\text { Posterior laryngitis } \\
\text { Disorder in vocal fold epithelium }\end{array}$ & LFR \\
\hline 28 & M & Vocal change, chronic cough & Gastroenterology & Normal & NVCM \\
\hline 65 & $\mathrm{~F}$ & Vocal change, respiratory disorder, chronic cough & Gastroenterology & $\begin{array}{l}\text { Interarythenoid hyperplasia, } \\
\text { Disorder in vocal fold epithelium }\end{array}$ & LFR \\
\hline 53 & $\mathrm{~F}$ & Chronic cough & Gastroenterology & Posterior laryngitis & LFR \\
\hline 18 & $\mathrm{~F}$ & Vocal change, respiratory disorder, chronic cough & Gastroenterology & $\begin{array}{l}\text { Posterior laryngitis, } \\
\text { Interarythenoid hyperplasia }\end{array}$ & LFR \\
\hline 44 & M & Chronic cough & Gastroenterology & $\begin{array}{l}\text { Interarythenoid hyperplasia, } \\
\text { Disorder in vocal fold epithelium }\end{array}$ & LFR \\
\hline 26 & $\mathrm{~F}$ & Vocal change, respiratory disorder, chronic cough & Gastroenterology & Normal & NVCM \\
\hline 72 & $\mathrm{~F}$ & Vocal change, respiratory disorder, chronic cough & Gastroenterology & Normal & NVCM \\
\hline 65 & $\mathrm{~F}$ & Chronic cough & Gastroenterology & Normal & NVCM \\
\hline 47 & $\mathrm{~F}$ & Vocal change, respiratory disorder, chronic cough & Gastroenterology & $\begin{array}{l}\text { Interarythenoid hyperplasia, } \\
\text { Disorder in vocal fold epithelium }\end{array}$ & LFR \\
\hline 38 & M & Vocal change, respiratory disorder, chronic cough & Gastroenterology & $\begin{array}{l}\text { Interarythenoid hyperplasia, } \\
\text { Disorder in vocal fold epithelium }\end{array}$ & LFR \\
\hline 31 & $\mathrm{~F}$ & Chronic cough & Gastroenterology & Posterior laryngitis & LFR \\
\hline 33 & M & Chronic cough & Gastroenterology & Posterior laryngitis & LFR \\
\hline 27 & $\mathrm{~F}$ & Vocal change, respiratory disorder, chronic cough & Gastroenterology & $\begin{array}{l}\text { Posterior laryngitis, } \\
\text { Interarythenoid hyperplasia }\end{array}$ & LFR \\
\hline 58 & $\mathrm{~F}$ & Vocal change, respiratory disorder, chronic cough & Gastroenterology & $\begin{array}{l}\text { Posterior laryngitis, } \\
\text { Interarythenoid hyperplasia }\end{array}$ & LFR \\
\hline 29 & $\mathrm{~F}$ & Vocal change, respiratory disorder & Gastroenterology & Posterior laryngitis & LFR \\
\hline 31 & $\mathrm{~F}$ & Vocal change, respiratory disorder, chronic cough & Gastroenterology & Normal & NVCM \\
\hline 48 & M & Respiratory disorder & Gastroenterology & Normal & NVCM \\
\hline 42 & $\mathrm{~F}$ & Vocal change, respiratory disorder, chronic cough & Gastroenterology & Normal & NVCM \\
\hline 29 & $\mathrm{~F}$ & Vocal change, chronic cough & Gastroenterology & $\begin{array}{l}\text { Posterior laryngitis, } \\
\text { Interarythenoid hyperplasia }\end{array}$ & LFR \\
\hline 23 & M & Vocal change, chronic cough & Gastroenterology & Posterior laryngitis & LFR \\
\hline 46 & $\mathrm{~F}$ & Vocal change, chronic cough & Gastroenterology & Posterior laryngitis & LFR \\
\hline 37 & M & Vocal change, respiratory disorder, chronic cough & Ear Nose Throat & Normal & NVCM \\
\hline 33 & $\mathrm{~F}$ & Vocal change, respiratory disorder, chronic cough & Ear Nose Throat & Normal & NVCM \\
\hline 34 & M & Vocal change & Ear Nose Throat & Disorder in vocal fold epithelium & LFR \\
\hline 63 & $\mathrm{~F}$ & Vocal change & Ear Nose Throat & Normal & NVCM \\
\hline 62 & $\mathrm{~F}$ & Vocal change, respiratory disorder, chronic cough & Ear Nose Throat & Normal & NVCM \\
\hline 32 & $\mathrm{~F}$ & Vocal change, respiratory disorder, chronic cough & Ear Nose Throat & Normal & NVCM \\
\hline 38 & $\mathrm{~F}$ & Vocal change & Ear Nose Throat & Left vocal cord paramedian fixation & Vocal cord paralysis \\
\hline 35 & $\mathrm{~F}$ & Respiratory disorder, episodic dyspnea & Psychiatry & Normal & NVCM \\
\hline 44 & M & Respiratory disorder, episodic dyspnea & Psychiatry & Normal & NVCM \\
\hline 47 & $\mathrm{~F}$ & Respiratory disorder, episodic dyspnea & Psychiatry & Normal & NVCM \\
\hline 37 & M & Vocal change, respiratory disorder, chronic cough & Psychiatry & Normal & NVCM \\
\hline 45 & $\mathrm{~F}$ & Respiratory disorder, inspiratory stridor & Psychiatry & Normal & NVCM \\
\hline 48 & M & Episodic dyspnea, inspiratory stridor & Psychiatry & Normal & NVCM \\
\hline 39 & $\mathrm{~F}$ & Respiratory disorder, episodic dyspnea & Psychiatry & Normal & NVCM \\
\hline 37 & M & Vocal change, respiratory disorder, chronic cough & Psychiatry & $\begin{array}{l}\text { Posterior laryngitis, } \\
\text { Interarythenoid hyperplasia }\end{array}$ & LFR \\
\hline 49 & $\mathrm{~F}$ & Respiratory disorder, episodic dyspnea & Psychiatry & Normal & NVCM \\
\hline 37 & $\mathrm{~F}$ & Respiratory disorder, episodic dyspnea & Psychiatry & Normal & NVCM \\
\hline 35 & $\mathrm{~F}$ & Episodic dyspnea & Psychiatry & Normal & NVCM \\
\hline
\end{tabular}

F: female, LFR: laryngopharyngeal reflux, M: male, NVCM: normal vocal cord motions, PVCM: paradoxical vocal cord motions. 
In conclusion, 'paradoxical vocal cord motions' are generally mistaken with asthma and misdiagnoses are made and faulty treatments are applied. Although PVCM is a rarely seen disease, it should be taken into consideration by the clinicians as it may lead to serious problems in non-diagnosed patients. The present study shows that PVCM can be diagnosed by provocative exercises performed at the time of the attack, not during asymptomatic period.

Conflict of Interest: No conflicts declared.

\section{References}

1. Matrka L. Paradoxic vocal fold movement disorder. Otolaryngol Clin North Am 2014;47:135-46.

2. Forrest LA, Husein T, Husein O. Paradoxical vocal cord motion: classification and treatment. Laryngoscope 2012;122:844-53.

3. Christopher KL, Wood RP 2nd, Eckert RC, Blager FB, Raney RA, Souhrada JF. Vocal cord dysfunction presenting as asthma. N Engl J Med 1983;308:1566-70.

4. Brugman SM. The many faces of vocal cord dysfunction. Am J Resp Crit Care Med 2003;167:A588.

5. Yilmaz A, Guven M, Eyibilen A, Aladag I. Paradoxical vocal fold motion dysfunction in asthma patients. Respirology 2009;14: 729-33.

6. Yelken K, Güven M, Aladağ I, Eyibilen A. Relations between symptoms, durations and triggers of attacks and concurrent diseases in paradoxical vocal fold movement disorder. [Article in Turkish] Kulak Burun Bogaz Ihtis Derg 2009;19:16-21.

7. O'Connell M. Vocal cord dysfunction: ready for prim-time? Ann All Asthma Imm 2006;96:762-3.

8. Mathers-Schmidt BA. Paradoxical vocal cord motion: a tutorial on a complex disorder and the speech language pathologist's role. Am J Speech Lang Pathol 2001;10:111-25.

9. Christopher KL. Understanding vocal cord dysfunction: a step in the right direction with a long road ahead. Chest 2006;129:842-3.

10. Dogan S, Eryuksel E, Karakurt S. Paradoxical vocal cord adduction: a case report. Medscape Allergy Clin Immunol 2002;2:1-7.

11. Murkami Y, Kirchner J. Mechanical and physiological properties of reflexlaryngeal closure. Ann Otol 1972;81:59-72.

12. Jain S, Bandi V, Officer T, et al. Role of vocal cord function and dysfunction inpatients presenting with symptoms of acute asthma exacerbation. J Asthma 2006;43:207-12.

13. Kenn K, Willer G, Bizer C, et al. Prevalence of vocal cord dysfunction in patients with dyspnoea. First prospective clinical study. Am J Respir Crit Care Med 1997;155:A965.

14. Kenn K, Balkissoon R. Vocal cord dysfunction: what do we know? Eur Respir J 2011;37:194-200.
15. Brugman SM, Simon SM. Vocal cord dysfunction: don't mistake it for asthma. Physician Sports Med 1998;26:63-74.

16. Bahrainwala AH, Simon MR. Wheezing and vocal cord dysfunction mimicking asthma. Curr Opin Pulm Med 2001;7:8-13.

17. O'Connell MA, Sklarew PR, Goodman DL. Spectrum of presentation of paradoxical vocal cord motion in ambulatory patients. Ann All Asthma Imm1995;74:341-4.

18. Powell DM, Karanfilov BI, Beechler KB, et al. Paradoxical vocal cord dysfunction in juveniles. Arch Otolayngol Head Neck Surg 2000;126:29-34.

19. Ayers JG, Gabbott PLA. Vocal cord dysfunction and laryngeal hyperresponsiveness: a function of altered autonomic balance? Thorax 2002;57:284-5.

20. Selner JC, Staudenmayer H, Koepke JW, et al. Vocal cord dysfunction: the importance of psychological factors and provocation challenge testing. J Allergy Clin Immunol 1987;79:726-33.

21. Bahrainwala AH, Simon MR. Wheezing and vocal cord dysfunction mimicking asthma. Curr Opin Pulm Med 2001;7:8-13.

22. Newman KB, Mason UG, Schmaling KB. Clinical features of vocal corddysfunction. Am J Respir Crit Care Med 1995;152: 1382-6.

23. Jain S, Bnadi V, Zimmerman J, et al. Incidence of vocal cord dysfunction inpatients presenting to emergency room with acute asthma exacerbation. Chest 1999;116:243.

24. Ibrahim WH, et al. Paradoxical vocal cord disorder: past, present and future. Postgrad Med J 2007;83:164-72.

25. Heimdal JH, Roksund OD, Halvorsen T, Skadberg BT, Olofsson J. Continuous laryngoscopic exercise test: a method for visualizing laryngeal dysfunction during exercise. Laryngoscope 2006;116: 52-7.

26. Suttithawil W, Chakkaphak S, Jaruchinda P, et al. Vocal cord dysfunction with a nutcracker esophagus and the role of gastroesophageal reflux disease. Ann All Asthma Imm 2006;96:373-5.

27. Kisson N, Kornick JB, Frewen TC. Psychogenic upper airway obstruction. Pediatrics 1988;81:714-17.

28. Balkissoon R, Kenn K. Asthma: vocal cord dysfunction (VCD) and other dysfunctional breathing disorders. Semin Respir Crit Care Med 2012;33:595-605.

29. Ibrahim WH, Gheriani HA, Almohamed AA, Raza T. Paradoxical vocal cord motion disorder: post, present and future. Postgrad Med J 2007; 83: 164-72.

30. Karaman E, Duman C, Alimoglu Y, Isildak H, Oz F. Paradoxical vocal cord motion-haloperidol usage in acute attack treatment. J Craniofac Surg 2009;20:1602-4.

31. Garibaldi E, LeBlance G, Hibbett A, et al. Exercise induced paradoxical vocal cord dysfunction: diagnosis with videostroboscopic endoscopy and treatment with Clostridium toxin. J Allergy Clin Immunol 1993;91(1 Part 2):200.

This is an open access article distributed under the terms of the Creative Commons Attribution-NonCommercial-NoDerivs 3.0 Unported (CC BYNC-ND3.0) Licence (http://creativecommons.org/licenses/by-nc-nd/3.0/) which permits unrestricted noncommercial use, distribution, and reproduction in any medium, provided the original work is properly cited.

Please cite this article as: Yalçınkaya E, Güler İ, Kocatürk S. Evaluation of paradoxical vocal cord motion and differential diagnosis. J Med Updates 2014;4(3):99-104. 